\title{
Nordic Symposium on Cloud Computing and Internet Technologies (NordiCloud)
}

\author{
Muhammad Ali Babar \\ IT University of Copenhagen \\ Copenhagen, Denmark \\ (+45) 72185107 \\ malibaba@itu.dk \\ Parastoo Mohagheghi \\ NTNU, Trondheim \\ Norway \\ (+47) 73593440 \\ parastoo@idi.ntnu.no
}

\author{
Åke Edlund \\ $\mathrm{KTH} / \mathrm{CSC} / \mathrm{PDC}$ \\ Sweden \\ (+46) 706621510 \\ edlund@pdc.kth.se \\ Sébastien Mosser \\ SINTEF, Oslo \\ Norway \\ (+47) 22067633 \\ Sebastien.Mosser@sintef.no
}

\author{
Tommi Mikkonen \\ Technical University of Tampere \\ Finland \\ (+358) 408490749 \\ tjm@cs.tut.fi \\ Peep Küngas \\ University of TarTu \\ Estonia \\ (+372) 7375445 \\ peep.kungas@soatrader.com
}

\begin{abstract}
This is an introduction to the NordiCloud Symposium collocated with WICSA/ECSA 2012.
\end{abstract}

\section{Categories and Subject Descriptors \\ A.0 [General]: Conference Proceedings}

\section{General Terms}

Measurement, Performance, Design, Security, Standardization.

\section{Keywords}

Cloud Computing, Utility Computing, Software Services, Internet of things (IoT), SaaS, PaaS, IaaS.

\section{INTRODUCTION}

Cloud Computing is an emerging computing paradigm, which promises to be a major driver of business innovation by exploiting the technological advances of Infrastructure as a Service (IaaS), Platform as a Service (PaaS), and Software as a Service (SaaS). Like in other parts of the World, many companies as well as public sector from Nordic and Baltic countries.

Given the increasing need and importance of knowledge of and skills in cloud computing, several research groups in Nordic/Baltic countries have started initiatives aimed at gaining in-depth understanding of the challenges involved and finding potential solutions for moving organizational resources, software and services on Cloud-enabled infrastructures. This situation

Copyright is held by authors/owners(s).

WICSA/ECSA 2012. August 20-24, 2012, Helsinki, Finland. ACM 978-1-4503-1568-5/12/08 provides an excellent opportunity to harness the growing cloud computing knowledge and expertise and the partners' existing networks to build an eco-system of cloud computing competency in Nordic and Baltic regions.

The Nordic Symposium on Cloud Computing \& Internet Technologies (NordiCloud) has been initiated with the main goal of providing an industrial and scientific forum for enhancing collaboration between commercial and research partners from Nordic and Baltic countries in the area of Cloud Computing and Internet Technologies. The target audience of the symposium are researchers and practitioners from Nordic/Baltic countries working in Cloud Computing or related areas of research and practice. This forum will provide an opportunity to participants to build synergies for levering existing and/or building new competencies for exploiting the Cloud Computing and Internet Technologies for improving the well-being of societies in general and the Nordic/Baltic regions in particular. This forum will be used to encourage the exchange of knowledge and experience and discuss future trends for Cloud Computing and Internet Technologies. This forum also aims to provide a bridge between the cloud computing researchers and practitioners from Nordic/Baltic countries and Cloud computing communicaties in other parts of the World.

\section{THE SYMPOSIUM THEMES AND ORGANIZATION}

\subsection{Main Themes for Contributions}

The symposium sought contributions on the topics related to cloud computing and Internet Technologies; some example topics for which we sought contributions were:

- Service and deployment models of Cloud computing

- Design and evaluation of systems for cloud-based technologies

- Service-oriented architectures

- Monitoring and adaptation challenges and solutions

- Software development paradigms for cloud computing 
- Evaluation approaches for cloud providers and cloudbased systems

- Energy efficiency of cloud based system and data centres

- Economic and business perspectives on cloud computing

- Socio-technical issues

- Internet-of-Things (IoT) for smart offices and homes

- cloud computing and Internet technologies for social computing

- Privacy and security issues and solutions

- Training and education programs for cloud computing

- Programming paradigms for cloud computing

- Cloud computing for supporting software development paradigms and organizational structures

\subsection{Organization}

The organization of the symposium was as follows:

General Chair

- $\quad$ M. Ali Babar, IT University of Copenhagen, Denmark.

Program Chairs

- $\quad$ Åke Edlund, KTH/CSC/PDC, Sweden.

- Tommi Mikkonen, Technical University of Tempera, Finland.

- Parastoo Mohagheghi, the Norwegian University of Science and Technology, Norway.

Symposium Coordination Chair

- Sébastien Mosser, SINTEF, Norway.

Publication Chair

- $\quad$ Peep Küngas, University of TarTu, Estonia.

Program committee

- $\quad$ Ajith Abraham, MIR Labs, USA

- Wolfgang Gentzsch, Rechenzentrum Garching (RZG), Germany

- Klaus Marius Hansen University of Copenhagen, Denmark

- Peter Kunzst, SystemsX.ch, Switzerland

- Pekka Lehtovuori, IT Center for Science, Finland

- Lars Lundberg, BTH, Sweden

- Tomi Männistö, Aalto University, Finland

- Mihhail Matskin, KTH, Sweden

- $\quad$ Eila Ovaska, VTT, Finland

- Jakob Illeborg Pagter, Alexandra Institute, Denmark

- Dana Petcu, Western University of Timisoara, Romania

- Ivan Porres, Åbo University, Finland

- Romain Rouvoy, INRIA Lille-Nord Europe, France
- Satish Srirama, University of Tartu, Estonia

- Sasu Tarkoma, University of Helsinki, Finland

- Johan Tordsson, Umeå University, Sweden

\section{Accepted Papers}

Following are the details along with the abstracts of the peerreviewed contributions accepted in all kinds of categories of NordiCloud Symposium.

Chauhan, M.A., Ali Babar, M., "Cloud infrastructure for providing tools as a service: Quality attributes and Potential Solutions"

This paper asserts that one of the domains, where cloud computing is expected to gain huge traction is Global Software Development (GSD) that has emerged as a popular software development model. The authors assert that providing Tools as a Service (TaaS) to GSD teams through a cloud-based infrastructure can be a promising solution to address the tools related challenges in GSD projects. Through an extensive review of the relevant literature on GSD and Cloud Computing, we have identified a set of quality attributes and potential architectural solutions for a cloud-based infrastructure that can provide TaaS to GSD teams. This paper outlines the promised benefits of TaaS to GSD teams, describes the expected quality attributes of a cloud based infrastructure and how these quality attributes can be achieved. We also present a reference architecture for a cloud based infrastructure to provide TaaS in GSD projects and describe its use with a detailed scenario of GSD projects.

Mikkonen, T., and Nieminen, A., "Elements for a cloud-based development environment"

This paper proposes a cloud based development environment. Authors claim that even today it is feasible to build a cooperative development environment that runs in a cloud, following the spirit of Google Docs, where developers can cooperate in real time. Authors take a step beyond from the design of the technical artifact that allows collaborative coding, and consider the consequences of such an approach to software development in a more general sense, in particular from the perspective of version management. The goal is to integrate collaborative development with traditional facilities commonly associated with software engineering. This in turn will lead to a development approach where new opportunities complement tools that are known to work and provide a lot of added value in software development.

Zhao L., Sakr S., Zhu L., Xu X., Liu A.,"An Architecture Framework for Application-Managed Scaling of Cloud-Hosted Relational Databases"

This paper reports architecture for scaling relational database in the cloud. Authors claim that it is important that applications can directly monitor fine-grained scaling performance (such as consistency-related replication delays and queryspecific response time) and specify application-specific policies for autonomic management of the scaling. This paper presents an architecture framework to facilitate a consumer-centric, applicationmanaged autonomic scaling of relational databases in cloud. The architecture framework includes a new consumer-centric monitoring infrastructure and customisable components for sensing, monitoring, analysing and actuation according to application-level scaling policies without modifying an existing application. 
Todoran I., Glinz M., "Towards Bridging the Communication Gap Between Consumers and Providers in the Cloud"

The paper reports a position motivated by the growing number of available cloud services and the challenge for consumers to choose the appropriate cloud services for their needs, whereas providers require dedicated ways to correctly elicit requirements from very heterogeneous consumers. Authors present the idea of StakeCloud community platform that can act as a cloud resources marketplace, allowing consumers to input their needs and providing them with matching cloud services.

\section{Neerbek J., "Message-driven FP-growth"}

The paper reports the work based on one of the best algorithms for doing frequent itemset mining is the known FP-growth (Frequent Patterns growth). We develop a cloud-enabled algorithmic variant for frequent itemset mining that scales with very little communication and computational overhead and even, with only one worker node, is faster than FP-growth. Authors report the concept of a postfix path and show how this allows them to lower the communicational cost and leads to adjustable work sizes. This concept provides a very flexible algorithmic solution that can be applied to a wide variety of different problem sizes and setups.

Laitinen J., Tourunen O., Sternkopf D., Laurikainen R., Öster P., Lehtovuori P.T., Miettinen T.A., Simonen T., Perheentupa T., Västrik I., Kallioniemi O., Lyall A., Thornton J., "Delivering ICT Infrastructure for Biomedical Research"

This paper describes an implementation of the Infrastructure-as-aService (IaaS) concept for scientific computing and seven service pilot implementations with requirements from biomedical use cases at the CSC - IT Center for Science. The key service design requirements were enabling the use of any scientific software environment the use cases needed to succeed, and delivering the distributed infrastructure ICT resources seamlessly with the local ICT resources for the scientist users. The service concept targets the IT administrators at research organisations and delivers virtualised compute cluster and storage capacity via private network solutions. The virtualised resources can become part of the local cluster as virtual nodes for sharing the same file system as the physical nodes if the network performance is sufficient.

Miranda J., Guillén J., Canal C., "Enough About Standardization, Let's Build Cloud Applications"

The heterogeneous solutions provided by different cloud vendors have led to application interoperability and migratability issues. Companies that consume cloud services are tied to a single cloud provider due to the high costs of migrating software in the cloud. The paper presents an approach for modeling and developing cloud applications, based on MDE and software adaptation techniques. The proposed solution is both cloud vendor and user friendly as it allows the former to freely define their own cloud policies, whilst users continue to be free to choose a cloud provider, even after the application has been developed.

Phaphoom N., Oza N., Wang X., Abrahamsson P., "Does Cloud Computing Deliver the Promised Benefits for IT Industry?"

This paper examines the perceived benefits of cloud computing from the cloud users' perspectives. Cloud users include users of $\mathrm{SaaS} / \mathrm{PaaS} / \mathrm{IaaS}$, ranging from end-users, to service developers and system administrators. Using content analysis techniques, we examine one of the major discussion forums of cloud computing.
Rather than offering a yes/no answer to the question posed, we offer five empirically drawn insights on the perceived benefits of cloud computing. It is proposed that the empirical insights gained are valuable to both researchers and practitioners. The value lies in a more holistic view on the promised benefits' landscape.

\section{Petcu D., Sandru C., "Towards Component-based Software Engineering of Cloud applications"}

Component-based architectures are natural for Cloud computing. Few current software development frameworks are exploiting this fact. A proof-of-the-concept implementation of an open-source Platform-as-a-service dedicated to the development of component-based applications is shortly presented in this paper.

Vasar M., Srirama S.N., Dumas M., "Framework for Monitoring and Testing Web Application Scalability on the Cloud"

This paper reports a framework to aid engineers in performance testing of Web applications and services. Authors have developed a framework that integrates several common benchmarking and monitoring tools. The framework helps performance engineers to test applications under various congurations and loads. Furthermore, the framework supports dynamic server allocation based on incoming load using a response-time-aware heuristics. We validated the framework by deploying and stress-testing the MediaWiki application. An experimental evaluation was conducted aimed at comparing the response-time-aware heuristics against Amazon Auto-Scale.

Wallom D., Turilli M., Taylor G., Hargreaves N., Martin A., Raun A., McMoran A., "myTrustedCloud: Trusted Cloud Infrastructure for Security-critical Computation and Data Management"

The typical use cases for public clouds involve non-business critical applications, particularly where issues around security of utilization of applications or deposited data within shared public services are binding requisites. In this poster, a use case is presented illustrating how the integration of Trusted Computing technologies into an available Infrastructure as a Service (IaaS) platform - Eucalyptus - allows the security-critical energy industry to exploit the flexibility and potential economic benefits of Cloud Computing for their business-critical applications.

Razavian M., Lago P., "A Lean and Mean Strategy for Migration to Services"

This paper presents the results of an interview survey on the migration strategies in industry. The purpose of this paper is twofold: 1) to discover the migration strategies that industrial practice adopts 2) to identify the benets of making such strategies explicit. Results of the survey have been analyzed in terms of migration activities and the available knowledge assets. As a result, we generalize the practice of industrial migration into a Lean \& Mean SOA migration strategy. In addition, the uses of the strategy pinpoint promising industry-relevant research directions.

Musil J., Musil A., Winkler D., Biffl S., "A First Account on Stigmergic Information Systems and Their Impact on Platform Development"

This paper uses stigmergy, a form of indirect communication and self-organization, as basis for framing certain groups of software services in order to propose an architectural concept of stigmergic information systems and describe identified key fields(actor base, 
architecture, software ecosystem) influencing them. Thus enabling the creation of better solution stack configurations needed to support effective and efficient development of such services

\section{The program}

The symposium will be held in two days on 20-21 August 2012, co-located with the Joint 10th Working IEEE/IFIP Conference on Software Architecture \& 6th European Conference on Software Architecture (WICSA/ECSA 2012) in Helsinki, Finland. The final program included a keynote given by Anna Liu, presentations of nine full papers, one position paper and two posters, presentations of two European research projects, two presentations by practitioners (one from industry and one from public sector), in addition to a tutorial. The full program is published in http://nordicloud.net. The two presentations by practitioners focused on applying cloud computing in Nordea as a large company in banking and finance in Nordic area given by Dmitri Guirenko and Sakari Palko, and a framework agreement for cloud computing services to the Swedish public sector given by Daniel Melin. The details about the program are as follows:

\section{Software Development \#1}

Clouds in Nordea Technology Strategy

Dmitri Guirenko \& Sakari Palko

Elements for a cloud-based development environment

Tommi Mikkonen \& Antti Nieminen

\section{Software Development \#2}

Cloud infrastructure for providing tools as a service: Quality attributes and Potential Solutions

Muhammad Aufeef Chauhan and Muhammad Ali Babar

\section{$\underline{\text { Tutorial }}$}

Building private cloud with Open Source Software for Scientific Environments

Zoran Pantic and M. Ali Babar

\section{Stories from the trenches}

Public Cloud computing for the public sector

Daniel Melin

Does Cloud Computing Deliver the Promised Benefits for IT Industry?

Nattakarn Phaphoom, Nilay Oza, Xiaofeng Wang and Pekka Abrahamsson

Delivering ICT infrastructure for biomedical research

Tommi Nyrönen, Jarno Laitinen, Danny Sternkopf, Olli Tourunen, Risto Laurikainen, Per Öster, Pekka Lehtovuori, Timo A. Miettinen, Tomi Simonen, Teemu Perheentupa, Imre Västrik, Olli Kallioniemi, Andrew Lyall and Janet M. Thornton

\section{Frameworks}

Framework for monitoring and testing web application scalability on the clouds

Martti Vasar, Satish Narayana Srirama and Marlon Dumas

An architecture framework for application managed scaling of cloud-based relational database

Liang Zhao, Sherif Sakr, Liming Zhu, Xiwei Xu and Anna Liu

\section{Architecture}

A first account on Stigmergic information systems

Juergen Musil, Angelika Musil, Dietmar Winkler and Stefan Biffl (Institute of Software Technology and Interactive Systems

Towards Bridging the Communication Gap Between Consumers and Providers in the Cloud

Irina Todoran and Martin Glinz

Note on mOSAIC' support for Component-based Software Engineering involving Cloud Services

Dana Petcu and Calin Sandru

myTrustedCloud: Trusted Cloud Infrastructure for Securitycritical Computation and Data Management

Matteo Turilli, David Wallom, Andrew Martin, Anbang Ruan, Gareth Taylor, Nigel Hargreaves and Alan Mcmoran

\section{$\underline{\text { Research to Practice }}$}

Towards a Cloud Modeling language: From REMICS to MODAClouds \& PaaSage projects

Sébastien Mosser

Enough about standardization, Let's build cloud applications

Javier Miranda, Joaquín Guillén, Juan Manuel Murillo and Carlos Canal

Message driven FP growth

Jan Neerbek

A lean and mean strategy for migration to services

Maryam Razavian and Patricia Lago

\section{Panel Discussion}

Cloud Computing for Sustainable Environment and Societies

\section{AKNOWLEDGEMENTS}

The help from Muhammad Aufeef Chauhan in preparing this introduction is greatly appreciated. M. Ali Babar's contribution was partly funded by the Danish Agency for Science, Technology and Innovation under the project "Next Generation Technology for Global Software Development", \#10-092313. 